\title{
Research on the Forming Mechanism of Group Doctor-Patient Conflicts in China Based on the Complex Social Networks
}

\author{
Haiyan Zhao ${ }^{1}$, Yuanqing $\mathrm{Lu}^{1}$ and Fangxing $\mathrm{Ye}^{2}$ \\ ${ }^{1}$ School of Public Administration, Nanjing University of Information Science \& \\ Technology, Nanjing 210044, China \\ ${ }^{2}$ School of Marxism, Hohai University, Nanjing 210098, China
}

\begin{abstract}
The complex social network theory is applied to solve the group doctor-patient conflict in present paper. Results showed that there were different interests in the evolution process of doctor-patient conflict. A complex network structure was developed around core interests and core nodes in the process of doctor-patient conflict. And this network structure can be reinforced under the law of "robustness" and "self organized criticality". With the probability of the occurrence of events, the model of social behavior will change suddenly and make the events out of control.
\end{abstract}

Keywords: group conflict; doctor-patient conflict; complex network; forming mechanism

\section{Progress in Research on Group Doctor-patient Conflicts}

Western countries first started to study on group doctor-patient conflicts. In 1950s, some research results of this topic have already appeared, but before 1950s, there were few people studying it. The reason is that at that time, people believed in a very ideal doctor-patient relationship, which the doctors were kind and patients were faithful; everything looked perfect [1]. However, as time went on, doctor-patient conflicts started to get worse. For example, according to a 1989 Gallup poll, $67 \%$ thought that doctors paid too much attention on making money, and $26 \%$ said that they didn't respect doctors, because in their mind, doctors only focused on earning money, but they weren't willing to spend time on patients [2]. The ideal image of ideal doctor-patient relationship broke up, and the relationship was damaged badly [3] .After 1950s, the studies of doctor-patient conflicts in the west were mainly concentrated on psychology, sociology and politics. Researchers apply these theories or the combination of these theories to systematically find out the patients' social roles, differences in communication patterns, information asymmetry, and also the exploratory research of the basic medical system. During this period of time, some systematical theories were gradually forming and developing, such as the patient-role theory [4], which clearly posed the doctor-patient recognized problem that were led by the role difference; medical situation communication pattern theory [5] which believes that people need to follow three principles to communicate under a medical situation, which are decreasing uncertainty, providing action basis and strengthening doctor-patient relationship. In the west, instead of studying on group doctor-patient conflicts directly, most western scholars prefer to illustrate it in some theories. For example, they use theory of social integration, social conflict theory, utilitarianism, equalitarianism, civil society, and mass violence theory to study mass affairs. The perspectives and achievements of these studies brought some effects on the domestic study. 
In recent years, domestic scholars have keen interest on this hot topic, doctor-patient conflicts. Researching results have emerged in large numbers. They are descriptive, elucidative, but also, static. The causes of doctor-patient conflicts are the most popular study topic. For example, Lei Chang and Zhang Siyuan [6] analyze the cognitive differences of doctor-patient conflicts. They found out that the cognitive differences of patients in the aspects of medical particularity, expectations, trust, responsibility and results can have a negative impact on the doctor-patient relationship. In addition to cognitive factors, most of the research is based on the perspectives of political science, sociology, laws, economics to analyze the causes of conflicts between doctors and patients. For instance, some existing research shows that the media coverage of medical disputes, especially the unfair reports, has deepened distrust of the hospital in patients' minds, resulting in exacerbation of doctor-patient conflicts [7]. The conflict between medical resources and the public needs is the basis of the doctor-patient conflicts [8]. It can be said that the imbalanced demand is a necessary element of the conflict between doctors and patients. As long as the allocation of social resources is still unequal, it is difficult to completely resolve the conflict. After the conflict, the fairly intervention of the authority can help rebuild the doctor-patient relationship, and can also promote harmonious development of medical orders [9]. Certainly, the communication mode between doctors and patients can also affect the relationship between doctors and patients from another aspect, and it is the basic skill of the daily maintenance of the doctor-patient relationship [10]. While these basic factors affecting the doctor-patient relationship, and they are the important causes of the conflicts between doctors and patients, but how do these conflicts change from sporadic, individual phenomenon to group events? This question relates to the affecting mechanism of group doctor-patient conflicts. The thoughts on this question include internal reflection within the health care industry, such as Fang Zhen's study on the forming mechanism of the group incidents from 5 aspects, including the background, internal and external motivation, relationship structure, and function in order to reduce and prevent mass doctor-patient conflicts. Many scholars also discussed the related contents when it comes to group events, but this part of the contents mostly scattered in the big topic of group events analysis.

Given the above, the current domestic analysis about group doctor-patient conflicts is static, and it always tends to focus on factors (e.g., cognition, resources, communication, civil rights, etc.). Actually, static analysis of these factors cannot clarify the interaction between the factors. Moreover, it cannot describe the dynamic process among these factors either. Group doctor-patient conflict is a process of gradually forming, upgrading and bursting out, which is a systematical process of complex interactions. People need to catch two basic elements when analyze this dynamic process. One is participating groups, which means that recognize who have involved in the formation of group events. The other one is the impetus; what impetus do participants have and how does the process affect the direction and strength of the impetus. Complex social network theory provides a good theoretical foundation framework to analyze these two issues, and next, the paper will begin the study based on this theoretical perspective. 


\section{Complex Social Network Perspective of the Group Doctor-patient Conflict}

Complex social network is an important emerging theory and tool in social networks research. It has applied the research measures of complex network dynamics to the social networks dynamics, and has become not only the trend of the current study of social networks, but also a new angle of researching group events [11]. Typically, the social network means the relatively stable relationship formed by interact and contact among the society members, which acts directly on human social behavior. The socalled complex social network is a kind of abstract description of complex social systems. It not only has the common features of the general social networks, but also is more prominent in topological properties. Complex network is more of a mathematical tool, an available analytical method to solve the problem. Many real-world systems can be described by complex network. A network can be an abstract graph made of nodes and edges. If the network refers to the social network, then the nodes are the individual members of society under the particular social context, and the edges are social relations among social members. When take the information of nodes and edges out, it can form a complete complex network. By the end of the 20th century, the development of modern network science has brought great progress to help scholars understand the complex systems. "Small world" effect (the network nodes have a small average shortest path length between each other, varying typically as the logarithm of the number of nodes), and "scale-free networks" (many degree distribution of the actual networks can be fit with the power law distribution) have been discovered after that [12]. In the network, the distance between two points is the number of the edges in the shortest path connecting the two points. Averaging all the distances of the node pairs can obtain the average distance of the network (the average path length). Meanwhile, there are two properties to measure network parameters, namely, degree distribution and clustering coefficient. In current complex social networks, community structure analysis and dynamic branch network evolution theory are the two most applied theories.

The ultimate goal of complex network research is to understand the operation mode of the network system, and to predict and control the network system behavior, which involves a very wide scope, such as the system of percolation, phase transitions, synchronization, network search, and navigation, etc., [13]. In addition to strong application of these theories, many phenomena in daily life have begun to use these theories, such as computer viruses spread on the wire, the prevalence of infectious diseases in the crowd, and rumors spread in society. In fact, transmission of many things can use the complex network theory. This kind of work has also been carried out now, such as in China, there have been a lot of studies on knowledge and technology diffusion, transmission of financial risks, network of new products and other aspects. Actually, as early as 1932, there were already social network-related research, and the research produced some excellent results [14]. In 1954, the term "social networks" has been formally proposed in studies. Then, it is widely used in sociology, psychology, management and other related fields. Therefore, this paper applied complex social network theory and methods to the study of the forming mechanism of group doctorpatient conflict in order to "break through the paradigm of social network analysis, and to promote the development of social networks in social relations, network infrastructure, and other aspects of the dynamics process"[11], and thus can discuss the dynamic forming process of the group doctor-patient incidents in depth, and to provide 
some references and recommendations to solve such problems. The group doctorpatient conflict as a very complex social problem, in its generation, evolution and formation process, transmission of information, generation of group behavior, and the group interaction, are all important problems which can be studied by the complex social network theory. Moreover, the dynamic features of complex social network theory are especially suitable for such issues. Chinese scholars have proposed to use complex social networks to analyze group incidents, but they didn' $t$ use it to analyze a specific social issues or social events. Present paper is an attempt in applying the complex social network theory to the particular social facts.

\section{Network of Group Doctor-Patient Conflict}

Community refers to the subgraph of cohesive nodes. There are many connections between nodes but relatively few connections between subgraphs. To put it simple, a large number of network nodes concentrate inside the community and interact frequently with each other, yet networks do not interact quite often between themselves. As shown in Figure 1, the network contains three communities, namely, A, B, and C. Close connections abound within the communities while much fewer connections exist among these three communities. In a social network, it is obvious that members or individuals within the three communities share the same attribute. They may have common interest, values, or some other features. There are also certain interest-related or other material connections among between these communities. Community structure reflects the modularity and heterogeneity of the network, which means that the real network is composed of many types of nodes. Studies on the structure of community network is will benefit the analysis of the constituents of social network, and therefore will provide grounds for the reciprocities between different groups.

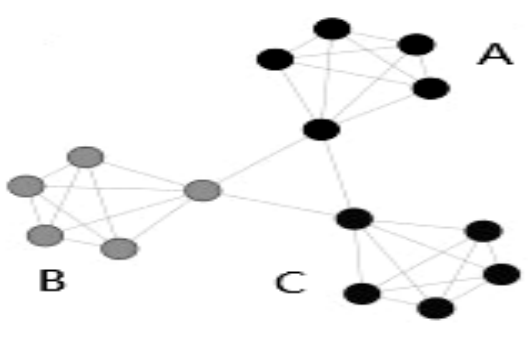

Figure 1

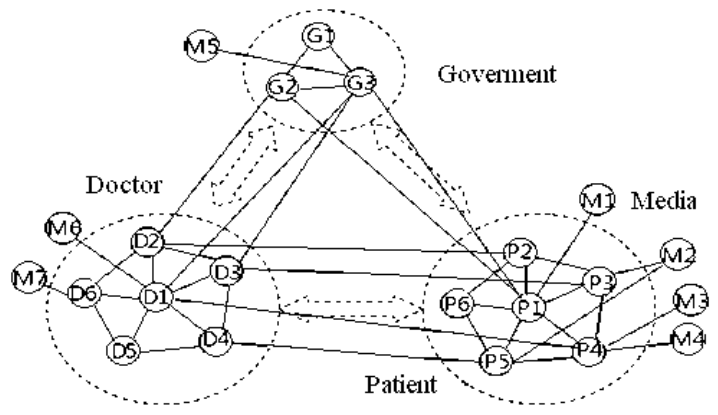

Figure 2

Based on this analysis framework, the structural differentiation of group doctor-patient conflict communities is done (Figure 2). Through the analysis of a group doctor-patient conflict caused by a child's death, we can discover that, there were different communities with certain interest demands involved in the process. These communities were, the patient's family members and relatives who had a direct claim of interest (P), the doctor community who had a direct defence of their own interest and vocational interest (D), government community who pursued social stability $(\mathrm{G})$, media community who indirectly sought both commercial and social interest (M). For communities directly involved in the conflict, each one of them (P, D, and $\mathrm{G})$ had a core of interest, and other relevant individuals were tightly connected centered on the mutual interest. Meanwhile, there was a central figure in each 
community, which was the central node in the community network. The community with indirect interest did not have a clear central nude. The individuals connected with each other in a loose way. Graph 2 is the community structure of a group doctor-patient conflict that happened in a children's hospital in Nanjing. In the conflict, the patient's family members and relatives wanted to maintain their own interest. The deceased child's parents were the core node, and the whole community was based on the core nude. P1, P2, P3, P4, P5, and P6 were used to indicate the members of the community. The doctor community was constituted by individual doctors and nurses, which were represented by D1, D2, D3, D4, D5, and D6. The government community was symbolized by G1, G2, and G3, which respectively stood for municipal party committee, health authorities, and police. This three-community structure is typical of today's group doctor-patient conflicts in China, and may serve as the base for further studies. What is worth noticing is that, due to the competition of interest and the looseness of connection among the media community, the following will not include detailed analysis of this community.

The differentiation of community structures does not only focus on interest communities, but also on the analysis of the core interest and core nodes of the community. The analysis of core nodes, in essence, is the analysis of influence in a community structure, or in other words, to seek out the most motivative and influential individual in the community. Generally speaking, there is a person at the center of each community. This person is the most influential one in his or her community and is termed core node. The core of the patient community is normally the immediate relatives of the patient (deceased or with aggravated symptoms). Their core interest usually covers the acquisition of financial compensation. The core of the doctor community is the doctor involved in the accident. As the conflict develops, the core node seems to transfer upward, towards the hospital management. The core interest of the doctor community at first is to protect the individual doctor involved in the accident. With the escalation of the situation, however, the core interest extends to the defence of the personnel and resources of the hospital even the vocational dignity of medical practitioners. With the extension of common interest, there is also an extension of the members inside the community, from those directly involved in the conflict to the whole personnel working in the hospital. The core of the government community also bears a feature of upward transfer. More and more government departments are involved in the conflict, and the core node keeps transferring upward. As for the doctor community and the government community, with the upward moving of the core node, their core interest changes gradually, and the motivations for settling group doctor-patient conflicts experience changes as well. The mentality of patching up a quarrel and reconciling the parties concerned begins to appear.

The interactions between communities (the directions of connections) also have obvious different characteristics. The patient community $(\mathrm{P})$ has direct conflict with the doctor community (D) but they seldom ask the government community (G) for help. This feature of connection are out of two reasons, the first one being that the patient community's interactions with the doctor community are often illegal and therefore they do not contact the police (G3), the second one being that the patient community distrust the health authorities (G2) based on their instincts and thus do not seek help from them, even hold strong antipathies against and vigilance upon them. The doctor community is the passive recipient of the patient community's irrational behavior, and is usually active in contacting the police. Their connection with the health authorities is bidirectional. In the government community, the police's core interest is the security and the stability of society while the health authorities' core interest lies in maintaining the proper rights of the doctors and the patients. 


\section{Network Dynamics of the Group Doctor-Patient Conflict}

The community network of the group doctor-patient conflict can help us understand the basic nodes and communities of the social network. But how does this structure develop? What are the factors affecting the changes of the structure? Is there interaction between the factors? Answers to these questions involve the network evolution dynamics of the group doctor-patient conflict. Currently there are two common types of studies on network dynamics, namely, robustness study, self-organized criticality study. Robustness is the system's ability to maintain its function when faced with changes in inner structure or outer environment, which means that robustness is a dynamic behavior of complex network evolution. Self-organized criticality means that in the evolutional process of social network, node distribution generally follows a smooth format despite the appearance of certain incidents with probability and regular patterns. The following will analyze the dynamic evolution of the doctor-patient conflict based on these principles.

On one hand, in a group doctor-patient conflict, the evolution of the community of the patient's family members and relatives clearly takes on robustness. The patient community is the most active community throughout the process. It propels the whole conflict to take place and changes. As stated in previous paragraphs, this community features many nodes surrounding the core node. Members of the community will experience several alterations. Sometimes new members join; sometimes old members quit. But these nuances will not affect the function of the community, which means that, once the patient community is formed, the behavior pattern to eliminate group doctor-patient conflict by restricting certain family members' radical behavior via coercive administrative methods $(G)$ is virtually ineffective. The robustness of communities allows for the absence of members, but the absence will not facilitate the decomposition of the community, nor will it facilitate the dysfunction of the community. Even certain members of the patient community are to some extent restrained, the community itself has a kind of impetus that facilitates the new situation of changed internal structure and external environment. It is of great importance for government to realize that coercive restrictive methods have limited effects and thus should be avoided.

On the other hand, self-organized criticality is typical in the patient community's behavior. This dynamic feature means the inevitability of irrational events with small probability. In the evolutional process of social network, the appearance of an individual event follows a rule, where there exists a function between its scale and its frequency. Many complex systems' behavior characteristics are classified as subcritical, critical, and supercritical states. In the normal state, the system will naturally evolve towards the critical state, yet when a operational system mutation happens, the system may reach the supercritical state and may continuously take on the massive avalanche phenomenon [15]. In the doctor-patient conflict, the patient community interacts frequently with the doctor community and the government community. With the increase of numbers of the members of the doctor community and the patient community and the exchange of information, natural evolution takes place. This kind of evolution makes the two communities evolve naturally towards the critical state. Normally, the critical state will remain unchanged, and there is a certain probability that the community behavior will experience an operational system mutation, and hence come to the avalanche of both parties' rational behavior patterns. As long as there forms a developed community structure between the doctor community and the patient community, it is certain that the behavior pattern in the interaction of the two parties has a certain probability to mutate, and lead to dramatic and unpredictable consequences. From the past doctor-patient conflict cases available (such as the one that happened in a children's hospital in Nanjing), we can come to the conclusion that there is a higher probability of the patient community to mutate. The mutation may be caused by a member's sudden irrational behavior, or by external forces 
(such as "professional" medical trouble maker). The consequences of the patient community's radical behavior pattern (such as medical personnel's getting hurt or humiliated) are very likely to incur the mutation of the doctor community's behavior pattern as reaction.

\section{Conclusions}

On the basis of previous studies, using complex social network theory, and focusing on the structure of the core node of the formation and evolution of group doctor-patient conflict, the paper systematically analyzed the forming mechanism of group doctor-patient conflict. Forming process of group doctor-patient conflict is extremely complicated. It is above all the interaction based on community structure. Each community has its own core node and the core benefit, and the forming process of conflict is the game process of different interest groups. It has its own rules, and it cannot be limited or eliminated forcefully. Robustness makes patient associations have the stability and continuity, and some members are missing or have limitations, but this cannot limit the patient's core functions and pursuit of interests; it has a conflict of "probability". Once the patient group and the doctor group formed together, the two sides behavioral patterns are intrinsically probabilistic to have "avalanche". Simply, the forming process of the doctor-patient conflict is in nature the gradual formation of patient's family or physician group. These groups, once formed, can have continuity and integrity. One-sided and high-pressure control measures of government cannot produce good results, cannot prevent well the brewing and mutation of mass doctor-patient conflicts, and may even stimulate, and promote patient' s family group to act out of control to stimulate the appearance of "avalanche". Certainly, the participation of some accidental factors (a radical member) and external forces (medical troubles) will promote patients' family or physician group cannot control their own behaviors. From the process of evolution, if the government wants to prevent mass doctor-patient conflicts, the most important thing lies in preventing the formation of patients' group. This requires the government to be able to provide unimpeded official channels to the families of patients and doctors' interest groups, and through multicommunity-participatory approaches to ensure the fairness of canal operation, so as to realize the harmonious coexistence of both doctors and patients and harmonious social relations.

\section{Acknowledgements}

This work was supported by Jiangsu Province preventive medicine project (Y2012060), supported by Jiangsu Health Department.

\section{References}

[1] D. Zhizheng, Medical Ethics Dictionary, Zhengzhou University Press, (In Chinese), (2003).

[2] W. C. Cockerham, "Medical Sociology", Prentice hall, (In Chinese), (2000).

[3] E. Shorter, "Doctors and their patients", New Brunswick, N. J., Transaction, (1991).

[4] T. Parsons, "The social system", Glencoe:The Free Press, (1951).

[5] E. J. Cassel, "Talking with patients", Combridge, Mass: MIT Press, vol. 2, (1985).

[6] C. Lei and S. Zhang, "A Survey Analysis of Cognitive Differences about Patients. Responsibility in Medical Disputes", Medicine and Philosophy( Humanistic \& Social Medicine Edition), (In Chinese), vol. 5, (2009), pp. 39-41.

[7] W. Wang, "Media's Social Responsibility in Reporting of Doctor-patient Conflicts", Medicine and Philosophy, (In Chinese), vol. 8A, (2012), pp. 22-24.

[8] G. Zhou, "Multiple Research and Thinking on Doctor-patient Dispute", The Chinese Health Service Management, (In Chinese), vol. 9, (2012), pp. 662-665.

[9] D. Jiang, "The Authority Should Promote the Trust between Doctor and Patient", Exploration and Free Views, (In Chinese), vol. 8, (2012), pp. 12-14. 
[10] Y. Chen, "Rhetoric of the Talk Between Doctor and Patient", Southeast Academic Research, (In Chinese), vol. 4, (2012), pp. 274-280.

[11] D. Wangi and L. He, "Complex Social Networks: A New Research Perspective on Formation Mechanism of the Group Incidents", Chinese Public Administration, (In Chinese), vol. 06, (2012), pp. 71-75.

[12] Z. Deng and W. Gan, "Community Structure Detection in Complex Net works", Computer Science, (In Chinese), vol. 6, (2012), pp. 103-108.

[13] T. Liu, Z. Chen and C. Xiaorong, "A Brief Review of Complex Networks and Its Application", Systems Engineering, (In Chinese), vol. 6, (2005), pp. 1-7.

[14] S. P. Borgatti, A. Mehra, D. J. Brass and G. Labianca, "Network Analysis in the Social Sciences", Science, vol. 323, no. 892, (2009).

[15] K. Mei, "Methodological Reflection on Application of Self-organized Criticality to Economics", Journal of System Science, (In Chinese), vol. 4, (2007), pp. 43-47.

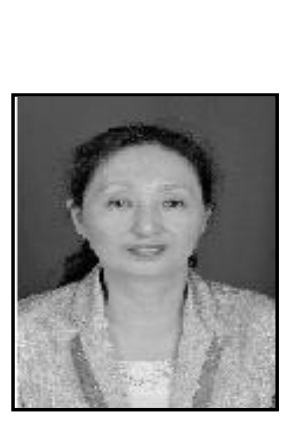

\section{Authors}

Haiyan Zhao, obtained her Bachelor degree in Mechanical Engineering from the South China Agricultural University in 1982. Now she is a professor in Nanjing University of Information Science and technology. Her research interest includes management science and engineering, specifically in fields of organization communication and network.

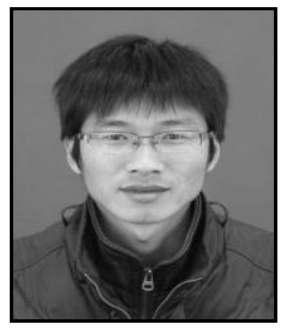

Yuanqing Lu, obtained his Bachelor degree in Education from Henan University in 2002 in China, Master degree in Science from Nanjing Normal University in 2005 in China. He received his Doctor in Management from Hohai University in 2013 in China. Now he is a associate professor in Nanjing University of Information Science and technology. His research interests mainly include management science and engineering.

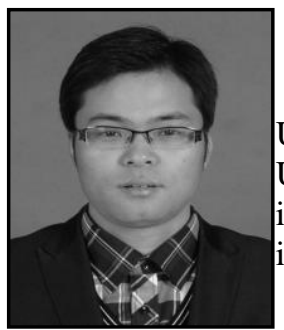

Fangxing Ye, obtained his Bachelor degree in Law from West Anhui University in 2007 in China, Master degree in Philosophy from Nanjing University of Information Science and Technology in in 2010 in China. He now is a PhD Candidate in School of Marxism, Hohai University, China. His research interests mainly include social management and organization network. 